\title{
26010 - POST-OPERATIVE DELIRIUM MONITORING BY THE ACUTE PAIN SERVICES
}

\section{Zeev Friedman MD, Jing Qin, ; Rita Qin, MD; Mount Sinai Hospital, Toronto, ON, Canada}

Introduction: Delirium is an acute disturbance in cognitive status involving fluctuating consciousness and significant inattention. Post-operative delirium in hospitalized patients has been linked to increased morbidity, mortality and length of stay1. The Acute Pain Service (APS) collectively monitors surgical patients on a daily basis and is therefore in a unique position to assess for delirium in this population. The purpose of this study was to assess the feasibility and effectiveness of routine screening for delirium by the APS using a modification of the Confusion Assessment Method (CAM) instrument. The CAM-ICU is a more concise adaptation of the CAM, the most widely-used instrument for assessing delirium by non-psychiatrists 2 .

Methods: This study was approved by the Mount Sinai Ethics Board. Patients undergoing surgery requiring more than 2 days of hospital stay were recruited over a period of 3 months. Each patient was assessed daily for 2 days after surgery for delirium using the CAM-ICU. Patients were also assessed for orientation to person, place and time, and for level of pain using a verbal analog scale out of 10. Information about the surgical procedure, medications, past medical history, anemia, electrolyte status and any diagnosis of confusion made by the medical or nursing staff were gathered from patients' charts. Results: 145 patients consented and were recruited for the study, with a mean age of 52.2 years. The incidence of delirium within 2 days after surgery was $7.6 \%$. Of these, $64 \%$ were disoriented to name, place or date. Only $18 \%$ of the patients diagnosed with delirium were noted as being confused by the medical or nursing staff. Each patient interview took an average of $4.9 \pm 0.4 \mathrm{~min}(95 \% \mathrm{Cl})$, although this increased to $9.05 \pm$ 2.0 min for delirious patients.

Discussion: In this study, routine screening of APS patients using the CAM-ICU detected more patients with post-operative delirium than did the standard nursing assessments of orientation in the 3 planes or the routine medical rounds. Adding the CAM-ICU to APS rounds would be a feasible endeavor as it requires little training, very basic materials, and only a few minutes per patient. The study interviews took an average of $4.9 \mathrm{~min}$, but since they also included an introduction to the study and the assessments of orientation and pain, the time required for adding just the CAM-ICU screening tool would be significantly shorter. The results of this pilot study show the use of the CAM-ICU as a routine APS monitor to be both feasible in its time consumption and valuable in its yield. Early detection of delirium may help in initiating prompt treatment, eliminating known risk factors and thus reducing morbidity.

References:

1.Crit Care. 2005 R: 375-81. Epub

2.JAMA 2001 286: 2703-2710 